\title{
Estrategias de marketing en sitios web de marcas de alimentos y bebidas consumidos por niños, niñas y adolescentes en la Argentina
}

\author{
Brand website marketing strategies for foods and beverages consumed by children and \\ adolescents in Argentina
}

\author{
Lic. Paula Gómez ${ }^{a}$ Lic. Camila Tamburini ${ }^{a}$, Lic. Vanesa Rodríguez García ${ }^{a}$ Lic. Verónica Chamorro y \\ Dr. Esteban Carmuega $a^{a}$
}

\begin{abstract}
RESUMEN
Internet permitió expandir la promoción de alimentos / bebidas a diferentes medios. El objetivo del estudio fue identificar las estrategias de marketing en sitios web de marcas de alimentos y bebidas consumidos por niños / as y adolescentes, y clasificarlos según las recomendaciones de las Guías Alimentarias para la Población Argentina.

Se seleccionaron los alimentos/bebidas más consumidos por la población infantojuvenil según la Encuesta Alimentaria Nutricional de la Ciudad Autónoma de Buenos Aires. Se identificaron las páginas web de las marcas; se analizaron las estrategias de marketing y se clasificaron los alimentos / bebidas promocionados según las Guías.

Se encontraron 80 páginas web, donde las estrategias más frecuentes fueron identificación de la marca, beneficios y declaraciones, y vínculos a páginas web y/o redes sociales. El $30 \%$ de los sitios estaban dirigidos a niños/as/adolescentes, donde el $75 \%$ de los alimentos/bebidas promocionados correspondieron al grupo de dulces y grasas, que incluye aquellos de bajo valor nutricional.

Palabras clave: mercadotecnia, páginas web, alimentos y bebidas, niño, adolescente.
\end{abstract}

http: / / dx.doi.org/10.5546/ aap.2021.51

Texto completo en inglés:

http: / / dx.doi.org/10.5546/ aap.2021.eng.51

Cómo citar: Gómez P, Tamburini C, Rodríguez García V, Chamorro $\mathrm{V}$, Carmuega E. Estrategias de marketing en sitios web de marcas de alimentos y bebidas consumidos por niños, niñas y adolescentes en la Argentina. Arch Argent Pediatr 2021;119(1):51-55.

a. Centro de Estudios sobre Nutrición Infantil, Ciudad Autónoma de Buenos Aires.

Correspondencia:

Lic. Paula Gómez: paula.gomez@cesni.org.ar

Financiamiento: El presente trabajo de investigación fue realizado con financiamiento propio de la Institución.

Conflicto de intereses: Ninguno que declarar.

Recibido: 1-5-2020

Aceptado: 18-8-2020

\section{INTRODUCCIÓN}

Internet ha permitido expandir la promoción y publicidad a diferentes medios, como las páginas web, redes sociales, aplicaciones, juegos y vídeos en la web..$^{1-3}$ Numerosas investigaciones han demostrado que los alimentos/bebidas publicitados en los medios de comunicación son altos en energía, azúcares agregados y grasas totales, y que pueden ser uno de los factores asociados al aumento del sobrepeso y la obesidad infantil. ${ }^{4-7}$ En la Argentina, según la Segunda Encuesta Nacional de Nutrición y Salud (2018), el 20,7 \% de la población de 5 a 17 años presenta sobrepeso, y el $20,4 \%$, obesidad. ${ }^{8}$

La Organización Mundial de la Salud pone de manifiesto que las empresas utilizan las nuevas tecnologías de comunicación para promocionar alimentos a los niños / as / adolescentes de la región de Latinoamérica. ${ }^{9,10}$ En los últimos años, diversas investigaciones observaron el uso de estrategias de marketing específicas para dirigirse a esta población en las páginas web de empresas alimentarias. ${ }^{11-13}$ El marketing en estos medios se define como una actividad promocional que se caracteriza no solo por el uso de técnicas creativas, sino también por el análisis de datos de los usuarios de la web para maximizar el impacto. ${ }^{2}$

La evidencia en esta temática es limitada en la Argentina, al igual que en el resto de América Latina. ${ }^{14}$ El objetivo del estudio fue identificar las estrategias de marketing en sitios web de marcas de alimentos y bebidas consumidos por niños, niñas y adolescentes, y clasificarlos según las recomendaciones de las Guías Alimentarias para la Población Argentina.

\section{POBLACIÓN Y MÉTODOS}

Se realizó un estudio descriptivo y de corte transversal. Para orientar la búsqueda de páginas web que pudieran presentar publicidad orientada a niños/as/adolescentes, se seleccionaron los 
alimentos/bebidas consumidos por más del $5 \%$ de la población de 5 a 17 años según la Encuesta Alimentaria Nutricional de la Ciudad Autónoma de Buenos Aires (2011). Esta encuesta tomó una muestra probabilística por conglomerados de 1987 individuos, en los cuales se aplicó un recordatorio de 24 horas para registrar el consumo de alimentos / bebidas.
Para identificar las marcas comerciales disponibles de los alimentos/bebidas seleccionados para el análisis, se consultó el catálogo virtual de las principales cadenas de supermercado de la Ciudad Autónoma de Buenos Aires, y se los ordenó según la opción orden de relevancia. Se excluyeron aquellos alimentos / bebidas que no contaban con marcas comerciales (Tabla 1).

TABLA 1. Categorías de alimentos y bebidas evaluadas y número de marcas incluidas

\begin{tabular}{|c|c|c|}
\hline Alimento o bebida & Porcentaje de consumidores* & Número de marcas evaluadas \\
\hline Aceite de girasol & 76,9 & 5 \\
\hline Azúcar & 61,8 & 3 \\
\hline Huevos & 61,3 & 2 \\
\hline Leche entera fluida & 52,6 & 6 \\
\hline Gaseosas regulares & 45,6 & 12 \\
\hline Manteca & 33,1 & 3 \\
\hline Fideos & 31,8 & 11 \\
\hline Cacao en polvo & 30,8 & 3 \\
\hline Arroz & 28,2 & 4 \\
\hline Queso rallado & 26,8 & 3 \\
\hline Tomate en conserva & 26,1 & 5 \\
\hline Pollo & 25,4 & 3 \\
\hline Harina de trigo & 24,1 & 3 \\
\hline Jugo en polvo & 22,2 & 3 \\
\hline Leche parcialmente descremada fluida & 23,4 & 6 \\
\hline Queso pasta semidura & 22,8 & 3 \\
\hline Jamón cocido & 22,6 & 5 \\
\hline Queso pasta blanda & 20,5 & 5 \\
\hline Mayonesa & 17,3 & 5 \\
\hline Galletitas dulces simples & 17,1 & 15 \\
\hline Tapa para empanada & 15,2 & 2 \\
\hline Manzana & 14,9 & 4 \\
\hline Galletitas tipo agua & 14,7 & 9 \\
\hline Jugo dietético en polvo & 14,1 & 4 \\
\hline Banana & 13,9 & 3 \\
\hline Galletitas dulces rellenas & 13,5 & 17 \\
\hline Alfajores de chocolate & 12,9 & 13 \\
\hline Mozzarella & 11,5 & 3 \\
\hline Dulce de leche & 11,4 & 5 \\
\hline Aguas saborizadas & 11,0 & 6 \\
\hline Pan lacteado & 10,5 & 5 \\
\hline Yogur & 10,1 & 7 \\
\hline Salchichas & 8,4 & 5 \\
\hline Caramelos & 7,5 & 11 \\
\hline Chocolate & 7,5 & 13 \\
\hline Crema de leche & 7,2 & 4 \\
\hline Queso untable & 6,9 & 5 \\
\hline Gaseosa dietética & 6,9 & 12 \\
\hline Papas fritas industrializadas & 6,9 & 5 \\
\hline Cereales para desayuno azucarados & 6,8 & 14 \\
\hline Hamburguesa comercial & 6,7 & 4 \\
\hline Jugos industrializados & 6,2 & 5 \\
\hline Barras de cereal & 6,0 & 4 \\
\hline Galletitas dulces surtidas & 5,6 & 3 \\
\hline Postre de leche industrializado & 5,5 & 6 \\
\hline Aceite de oliva & 5,2 & 8 \\
\hline Leche chocolatada & 5,0 & 5 \\
\hline Prefritos de pollo & 5,0 & 4 \\
\hline Gelatina & 5,0 & 5 \\
\hline
\end{tabular}

* Datos obtenidos de la Encuesta Alimentaria Nutricional de CABA (2011). 
Para cada marca comercial seleccionada, se buscó en Google ${ }^{\circledR}$ si contaban con página web, utilizando la marca como palabra clave, restringiendo a páginas web de la Argentina y excluyendo los sitios web corporativos de las compañías alimentarias. Para analizar las estrategias de marketing, se adaptaron los modelos desarrollados en otros estudios. ${ }^{11,12}$ Las estrategias se clasificaron en ocho categorías: I) identificación de la marca; II) secciones y juegos; III) promociones; IV) personajes y celebridades; v) vínculos a páginas web y/o redes sociales; vi) beneficios y declaraciones; vII) registros y descargas, y VIII) protección y aspectos legales. Se identificaron en la página principal y hasta dos clics dentro de esta. Las páginas web se

TABLA 2. Estrategias de marketing utilizadas en las páginas web de alimentos y bebidas

\begin{tabular}{|c|c|}
\hline & $\begin{array}{l}\text { Total de páginas web } \\
\text { (n=80) } \\
\text { Número }(\%)\end{array}$ \\
\hline $\begin{array}{l}\text { Identificación de la marca } \\
\text { Presencia del logo de la marca } \\
\text { Presencia del envase del producto } \\
\text { Producto como fondo de la página } \\
\text { Presencia de variedades de otros productos de la marca }\end{array}$ & $\begin{array}{l}80(100 \%) \\
79(98,8 \%) \\
70(87,5 \%) \\
60(75 \%) \\
60(75 \%)\end{array}$ \\
\hline $\begin{array}{l}\text { Beneficios y declaraciones } \\
\text { Presencia de recetas } \\
\text { Información nutricional } \\
\text { Declaración de beneficio de la marca } \\
\text { Declaración nutricional } \\
\text { Información sobre alimentación saludable } \\
\text { Declaración sobre salud en general }\end{array}$ & $\begin{array}{l}71(88,8 \%) \\
47(58,8 \%) \\
41(51,3 \%) \\
29(36,3 \%) \\
28(35 \%) \\
13(16,3 \%) \\
11(13,8 \%)\end{array}$ \\
\hline $\begin{array}{l}\text { Vínculos a páginas web y/o redes sociales } \\
\text { Vínculo a las redes sociales de la marca (Facebook e/o Instagram) } \\
\text { Presencia de posteos de redes sociales (Facebook e/o Instagram) } \\
\text { Anuncio de televisión de la marca } \\
\text { Enlace a otras marcas de la misma empresa } \\
\text { Enlaces a otros sitios web de alimentos } \\
\text { Enlaces a sitios web no relacionados con alimentos }\end{array}$ & $\begin{array}{l}68(85 \%) \\
65(81,3 \%) \\
16(20 \%) \\
11(13,8 \%) \\
9(11,3 \%) \\
3(3,8 \%) \\
0(0 \%)\end{array}$ \\
\hline $\begin{array}{l}\text { Protección y aspectos legales } \\
\text { Información legal } \\
\text { Condiciones de uso y cookies } \\
\text { Consentimiento adulto } \\
\text { Bloqueo de la página por edad }\end{array}$ & $\begin{array}{l}46(57,5 \%) \\
46(57,5 \%) \\
\quad 4(5 \%) \\
0(0 \%) \\
0(0 \%)\end{array}$ \\
\hline $\begin{array}{l}\text { Registro y descargas } \\
\text { Registro en el sitio web } \\
\text { Venta en línea } \\
\text { Aplicaciones } \\
\text { Material educativo sin la marca } \\
\text { Material educativo con la marca } \\
\text { Beneficios para miembros } \\
\text { Imágenes para el dispositivo electrónico o para colorear }\end{array}$ & $\begin{array}{c}20(25 \%) \\
13(16,3 \%) \\
7(8,8 \%) \\
2(2,5 \%) \\
2(2,5 \%) \\
1(1,3 \%) \\
1(1,3 \%) \\
0(0 \%)\end{array}$ \\
\hline $\begin{array}{l}\text { Personajes y celebridades } \\
\text { Presencia de dibujos animados } \\
\text { Presencia de personajes animados } \\
\text { Presencia de personas famosas o celebridades (televisión, radio, película, redes, música) } \\
\text { Presencia de deportistas }\end{array}$ & $\begin{array}{l}15(18,8 \%) \\
6(7,5 \%) \\
5(6,3 \%) \\
3(3,8 \%) \\
1(1,3 \%)\end{array}$ \\
\hline $\begin{array}{l}\text { Secciones y juegos } \\
\text { Sección exclusiva con contenido para niños/niñas/adolescentes } \\
\text { Juegos que incluyan la marca } \\
\text { Sección destinada a la familia } \\
\text { Juegos sin promoción de la marca }\end{array}$ & $\begin{array}{c}11(13,8 \%) \\
8(10 \%) \\
5(6,3 \%) \\
2(2,5 \%) \\
0(0 \%)\end{array}$ \\
\hline $\begin{array}{l}\text { Promociones } \\
\text { Concursos } \\
\text { Regalos } \\
\text { Recaudación de fondos con fines benéficos }\end{array}$ & $\begin{array}{c}11(13,8 \%) \\
7(8,8 \%) \\
4(5 \%) \\
1(1,3 \%)\end{array}$ \\
\hline
\end{tabular}


consideraron como dirigidas a niños/as / adolescentes cuando utilizaban estrategias como personajes o dibujos animados, famosos, juegos, aplicaciones, concursos o eventos destinados a atraer a dicho público, apelando a temáticas como diversión, aventura, fantasía, moda. ${ }^{12}$

Se clasificaron los alimentos/bebidas con página web según los grupos de las Guías de la siguiente forma: I) verduras y frutas; II) legumbres, cereales, papa, pan y pastas; III) leche, yogur y quesos; IV) carnes y huevos; v) aceite, frutas secas y semillas, y vi) dulces y grasas. ${ }^{15}$ Los primeros cinco grupos se recomiendan consumir diariamente por su aporte de nutrientes. El grupo dulces y grasas incluye alimentos/bebidas con elevado contenido de grasas, azúcares y sodio, por lo que se sugiere limitar su consumo.

Se diseñó un formulario de Google ${ }^{\circledR}$ para el registro de los datos y se implementó una prueba piloto. La recolección de datos fue durante agosto y septiembre de 2019. Para el análisis estadístico, se utilizó el programa Statistical Package for the Social Sciences versión 20 para Windows.

\section{Consideraciones éticas}

Todos los aspectos involucrados en el desarrollo de este proyecto se realizaron adhiriendo a las normativas vigentes nacionales e internacionales.

\section{RESULTADOS}

Se seleccionaron 195 marcas comerciales de alimentos / bebidas (Tabla 1). El $41 \%(\mathrm{n}=80)$ de las marcas contaban con página web, de las cuales 24 estaban dirigidas a niños/as / adolescentes.

En el total de páginas web analizadas, la categoría identificación de la marca fue la estrategia más utilizada (el $100 \%$ de las páginas), dentro de la cual el $99 \%$ contaba con la presencia de logo de la marca, y el $88 \%$, con la del envase. En segundo lugar, se observó la estrategia beneficios y declaraciones (el $89 \%$ ), dentro de la cual las más frecuentes fueron la presencia de recetas (el $59 \%$ ) y la información nutricional (el $51 \%$ ). En tercer lugar, se encontraron vínculos a páginas web y/o redes sociales, donde la estrategia más utilizada fue el vínculo a las redes sociales de la marca (el $81 \%$ ). Además, la categoría protección y aspectos legales estuvo presente en el $58 \%$ de los sitios web. En menor proporción, se encontró la categoría registros y descargas (el $25 \%$ ), y personajes y celebridades (el $19 \%$ ). Las estrategias menos utilizadas fueron las correspondientes a promociones (el $14 \%) \mathrm{y}$ secciones y juegos (el 14\%) (Tabla 2).

Los alimentos/bebidas promocionados en las páginas web se clasificaron de acuerdo con las Guías. El $63 \%$ pertenecían al grupo de dulces y grasas, seguido por el grupo de leche, yogur y quesos (el $13 \%$ ), legumbres, cereales, papa, pan y pastas (el $10 \%)$, verduras y frutas (el $8 \%)$, aceite y semillas (el $5 \%$ ), y carnes y huevos (el $1 \%$ ). $\mathrm{Al}$ analizar los alimentos/bebidas en los sitios dirigidos a niños/as/adolescentes, se observó una mayor proporción del grupo de dulces y grasas (el $75 \%)$, entre los que se encontraron las bebidas azucaradas, leche chocolatada, galletitas dulces, golosinas, cereales azucarados, postres de leche y salchichas. En segundo lugar, se encontró el grupo de leche, yogur y quesos (el $11 \%$ ), seguido por legumbres, cereales, papa, pan y pastas (el $7 \%$ ), aceites y semillas (el $4 \%$ ), y frutas y verduras (el $4 \%)$.

\section{DISCUSIÓN}

En la Argentina, las estrategias de presencia del logo y del envase fueron las más frecuentes. Esto coincidió con los datos proporcionados por Kelly y col., a partir de una revisión de 119 sitios web de las empresas alimentarias de Australia ${ }^{11}$ y con los resultados de una investigación que analizó 70 páginas web de alimentos/bebidas de Nueva Zelanda. ${ }^{12}$ Además, en Australia, se utilizaron frecuentemente las estrategias beneficios de la marca y la descarga de material educativo. ${ }^{11}$ El estudio de Weber y col. encontró que las páginas web estadounidenses utilizaban con mayor frecuencia registro y descargas, promociones, juegos y presencia de personajes. ${ }^{13}$ Dichas estrategias se utilizaron con menor frecuencia en las páginas web de la Argentina.

El porcentaje de páginas que dirigían sus estrategias a niños/as/adolescentes en la Argentina fue del $30 \%$, similar a la investigación de Nueva Zelanda, donde el $34 \%$ de los sitios estaban dirigidos a esta audiencia. ${ }^{12}$ En la investigación de Kelly y col., este valor fue del $13 \% .^{11}$

Al analizar los alimentos/bebidas promocionados según las Guías, se observó una mayor presencia de aquellos con alto contenido de grasas, azúcares y sodio, principalmente, en las páginas web dirigidas a niños/as/adolescentes. Esto fue coincidente con los resultados de otras investigaciones. El $61 \%$ en Australia ${ }^{11}$ y el $75 \%$ en Nueva Zelanda ${ }^{12}$ correspondieron a la categoría no saludable según los estándares del Ministerio 
de Salud de cada país.

La principal fortaleza del estudio es que aporta datos inéditos, ya que es el primero de estas características realizado en el país. Sin embargo, el mundo virtual tiene una dinámica que obliga a evaluar tendencias y ampliar el campo de investigación a otros medios digitales, como redes sociales, juegos virtuales y páginas web de uso frecuente en niños/as/adolescentes. Una debilidad del estudio es que, al no poder evaluar el tráfico en páginas web, carece de información cuantitativa para medir la exposición real al marketing de alimentos/bebidas.

La utilización de estrategias de marketing dirigidas a niños/as/adolescentes en el mundo virtual merece una discusión amplia en nuestra sociedad. Especialmente, cuando los alimentos/ bebidas promocionados son de baja calidad nutricional, según los resultados de este estudio.

\section{CONCLUSIÓN}

Se observó que 24 de las 80 páginas web analizadas utilizaban estrategias dirigidas a captar al público de niños / as / adolescentes, en las que 7,5 de cada 10 alimentos/bebidas promocionados fueron de bajo valor nutricional.

\section{Agradecimientos}

Los autores agradecen a la Dra. María Elisa Zapata y a la Bioq. Alicia Rovirosa por sus valiosos aportes en la revisión del documento.

\section{REFERENCIAS}

1. Regional Office for Europe of the World Health Organization. Marketing of foods high in fat, salt and sugar to children: update2012-2013. Denmark: WHO;2013. [Acceso: el24 de abrilde2020]. Disponibleen: http: / / www. euro.who.int/__data/ assets / pdf_file/0019/191125/ e96859.pdf.

2. Regional Office for Europe of the World Health Organization. Tackling food marketing to children in a digital world: trans-disciplinary perspectives. Copenhagen, Dinamarca: WHO, 2016. [Acceso: el 20 de junio de 2020]. Disponibleen:https: / / www.euro.who.int/_data/assets / pdf_file/0017/322226/Tackling-food-marketing-childrendigital-world-trans-disciplinary-perspectives-en.pdf.

3. World Cancer Research Fund International. Building Momentum: lessons on implementing robust restrictions of food and non-alcoholic beverage marketing to children. 2020. [Acceso: el5 dejulio de 2020]. Disponible en: https: / / www.wcrf.org/sites / default/files / PPA-BuildingMomentum-3-WEB-3.pdf.
4. Cairns G, Angus K, Hastings G, Caraher M. Systematic reviews of the evidence on the nature, extent and effects of food marketing to children. A retrospective summary. Appetite. 2013; 62:209-15.

5. Organización Mundial de la Salud. Informe de la Comisión para acabar con la obesidad infantil. Génova: OMS, 2016. [Acceso: el 24 de abril de 2020]. Disponible en: https: / / apps.who.int/iris/bitstream/ handle/10665/206450/9789243510064_spa.pdf;jsessioni $\mathrm{d}=$ DAE6B5DA0C2C4A2587A7E1FD61487939? sequence $=1$.

6. Rovirosa A, Zapata ME, GómezP, GotthelfS, et al. Alimentos y bebidas publicitados en canales infantiles de Argentina: frecuencia, duración y calidad nutricional. Arch Argent Pediatr. 2017; 115(1):28-34.

7. Sadeghirad B, Duhaney T, MotaghipishehS, CampbellNR, et al. Influence of unhealthy food and beverage marketing on children's dietary intake and preference: a systematic review and meta-analysis of randomized trials. Obes Rev. 2016; 17(10):945-59.

8. Argentina. Secretaría de Gobierno de Salud. Segunda Encuesta Nacional de Nutrición y Salud. Resumen Ejecutivo. Ministerio de Salud; 2018. [Acceso: el 20 de abril de2020]. Disponibleen: http:/ / www.msal.gob.ar/images / stories / bes / graficos / 0000001565 cnt-ennys2_resumenejecutivo-2019.pdf.

9. Organización Panamericana delaSalud. Recomendaciones de la consulta de expertos dela Organización Panamericana de la Salud sobre la promoción y publicidad de alimentos y bebidas no alcohólicas dirigida a los niños en la Región de las Américas. Washington, DC: OPS, 2011. [Acceso: el 24 de abril de 2020]. Disponible en: https: / / www.paho. org / hq / dmdocuments / 2012 / Experts-Food-Marketingto-Children-(SPA).pdf.

10. Organización Mundial de la Salud. Conjunto de recomendaciones sobrela promoción de alimentos y bebidas noalcohólicas dirigida a los niños. Suiza:OMS, 2010. [Acceso: el4 de abril de 2020]. Disponibleen: https: / / apps.who.int / iris / bitstream/handle/10665/44422/9789243500218_spa. pdf;jsessionid=78872EDFBE0C912DED76166671CDAF8A ?sequence $=1$.

11. Kelly B, Bochynska K, Kornman K, Chapman K. Internet food marketing on popular children's websites and food product websites in Australia. Public Health Nutr. 2007; 11(11):1180-7.

12. Vandevijvere S, Sagar K, Kelly B, Swinburn B. Unhealthy food marketing to New Zealand children and adolescents through the internet. N Z Med J. 2017; 130(1450):32-43.

13. Weber K, Story M, Harnack L. Internet food marketing strategies aimed at children and adolescents: a content analysis of food and beverage brand web sites. J Am Diet Assoc. 2006; 106(9):1463-6.

14. Chemas-Velez MM, Gómez LF, Velasquez A, Mora-Plaza $\mathrm{M}$, et al. Scoping review of studies on food marketing in Latin America:Summary of existing evidence and research gaps. Rev Saude Publica. 2019; 53:107.

15. Guías alimentarias para la población Argentina. Documento técnico metodológico. Buenos Aires: Ministerio de Salud de la Nación; 2016. [Acceso: el 28 de febrero de 2020]. Disponible en: http: / / www.msal.gob.ar/images/stories/ bes/graficos / 0000001007cnt-2017-06_guia-alimentariapoblacion-argentina.pdf. 